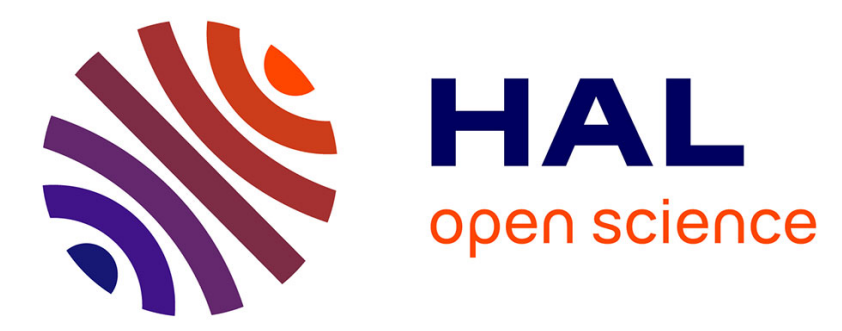

\title{
Caractéristiques optiques de miroirs électrostatiques à symétrie de révolution composés de trois cylindres de même rayon
}

C. Berger, M. Baril

\section{- To cite this version:}

C. Berger, M. Baril. Caractéristiques optiques de miroirs électrostatiques à symétrie de révolution composés de trois cylindres de même rayon. Revue de Physique Appliquée, 1981, 16 (4), pp.173-179. 10.1051/rphysap:01981001604017300 . jpa-00244910

\section{HAL Id: jpa-00244910 https://hal.science/jpa-00244910}

Submitted on 1 Jan 1981

HAL is a multi-disciplinary open access archive for the deposit and dissemination of scientific research documents, whether they are published or not. The documents may come from teaching and research institutions in France or abroad, or from public or private research centers.
L'archive ouverte pluridisciplinaire HAL, est destinée au dépôt et à la diffusion de documents scientifiques de niveau recherche, publiés ou non, émanant des établissements d'enseignement et de recherche français ou étrangers, des laboratoires publics ou privés. 


\title{
Caractéristiques optiques de miroirs électrostatiques à symétrie de révolution composés de trois cylindres de même rayon
}

\author{
C. Berger et M. Baril (*) \\ Laboratoire de Physique Industrielle, I.N.S.A. de Lyon, 69621 Villeurbanne Cedex, France \\ (*) Département de Physique et C.R.A.M., Université Laval, Québec, P. Qué., GIK 7P4, Canada
}

(Reçu le 18 juillet 1980, révisé le 9 décembre 1980, accepté le 13 janvier 1981)

\begin{abstract}
Résumé. - Une étude préliminaire des miroirs électrostatiques constitués de trois cylindres coaxiaux juxtaposés a montré que pour obtenir une évolution des paramètres optiques en fonction du rapport des potentiels extrêmes différente de celle des paramètres du miroir bicylindrique, il fallait un écartement suffisant des électrodes extrêmes. Deux géométries différentes sont retenues ici, pour lesquelles les propriétés optiques du premier ordre et du troisième ordre sont déterminées en fonction du rapport des potentiels extrêmes et du rapport des différences de potentiel entre cylindres. Deux séries de quatre tableaux donnent en fonction de ces variables : l'abscisse du sommet, le rayon du miroir, les coefficients d'aberration sphérique ou chromatique principale.
\end{abstract}

\begin{abstract}
A preliminary study of electrostatic mirrors made of three coaxial cylinders of equal radius has been made. The results show that if we want the optical properties to differ significantly from these of two coaxial cylinders of the same radius in terms of the ratio of end potentials, it is necessary to use a minimum spacing between the end cylinders in the order of the diameter of the cylinders. Two specific geometries have been studied. First and second order optical properties have been calculated in terms of the ratio of end potentials and of the ratio of the potential differences between the cylinders. Two series of four tables give, in terms of the preceding parameters, the following optical quantities : abscissa of the summit, radius of curvature of the mirror, principal chromatic and spherical aberrations.
\end{abstract}

1. Introduction. - Le développement de la spectrométrie de masse à multiples passages nécessitant l'utilisation de miroirs électrostatiques nous a conduits voici quelques années à nous intéresser aux miroirs électrostatiques.

Si la littérature fournissait à ce moment-là quelques valeurs concernant les propriétés optiques des miroirs formés par la juxtaposition de deux ensembles de deux plans parallèles [1]; il n'existait en revanche, à notre connaissance, aucune étude systématique sur les miroirs à symétrie de révolution.

Dans un premier temps nous avons donc développé le système le plus simple c'est-à-dire le miroir formé de deux cylindres coaxiaux juxtaposés de même rayon et de longueur suffisante pour être considérés comme infinis et nous en rappelons ici les caractéristiques du premier ordre (Fig. 1).

Nous pouvons voir que la zone en fonctionnement convergent correspond à un domaine étroit de valeur de $V_{2} / V_{1}$. Par ailleurs une étude succincte des aberrations au point de rebroussement [2] montre que l'utilisation de ce miroir ne paraît guère raisonnable en dehors de la zone du maximum de convergence.

De ce fait les investigations dans le domaine des miroirs à symétrie de révolution ont été poursuivies dans plusieurs directions. La façon la plus simple

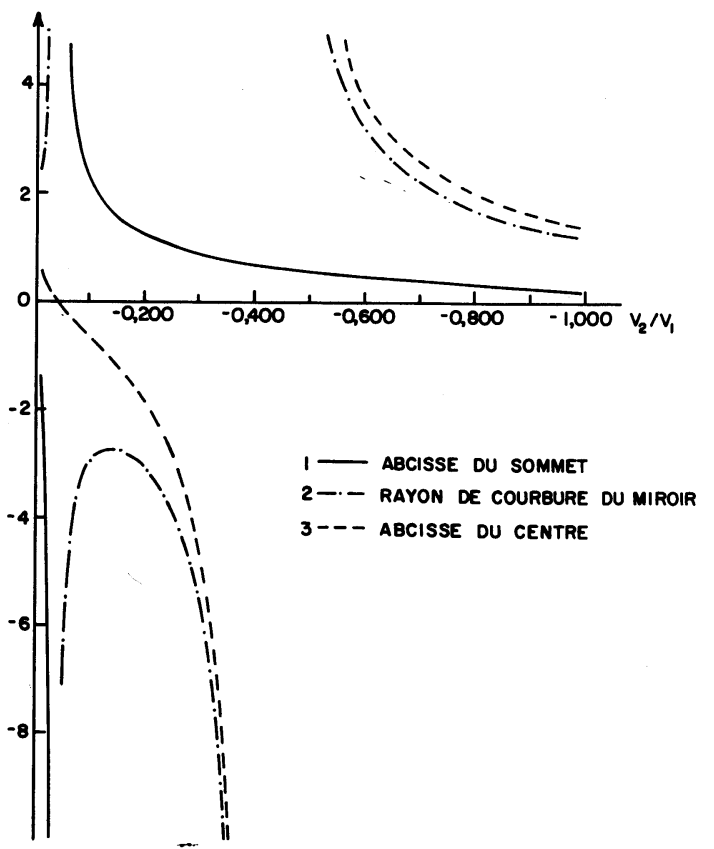

Fig. 1. - Caractéristiques optiques du miroir électrostatique à deux cylindres en fonction du rapport $V_{2} / V_{1}$ des potentiels appliqués aux cylindres.

[Optical characteristics of the bicylindrical electrostatic mirror with ratio $V_{2} / V_{1}$ between the potential applied to the cylinders.] 
d'obtenir une modification souhaitée dans l'évolution des caractéristiques des miroirs est de multiplier les facteurs géométriques et électriques. Nous présentons ici dans l'esprit de cette démarche une étude sur les miroirs à trois cylindres coaxiaux de même rayon. Le travail repose sur les deux hypothèses suivantes :

- les électrodes extrêmes sont suffisamment longues pour être traitées comme des cylindres de longueur infinie;

- dans les intervalles interélectrodes, le potentiel est supposé varier linéairement au niveau des cylindres.

Notons également qu'une étude récemment publiée [3] donne les propriétés focalisatrices d'un objectif de Johannson fonctionnant en miroir.

2. Choix des paramètres et nomenclature des miroirs étudiés. - Ces systèmes présentent plusieurs paramètres géométriques et électriques.

Le rayon $R$ des cylindres étant choisi comme unité de longueur, les deux variables géométriques retenues sont :

- la longueur $L$ séparant les deux cylindres extrêmes,

- la longueur $L_{\mathrm{e}}$ de l'électrode centrale, les intervalles interélectrodes étant choisis identiques et dénommés $e$ (Fig. 2).

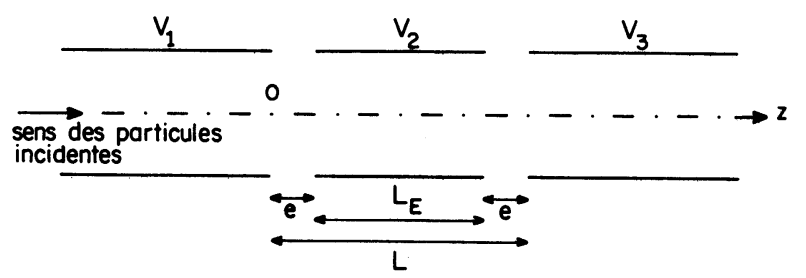

Fig. 2. - Miroir électrostatique à trois cylindres.

[Three cylinder mirror.]

Les potentiels des cylindres constituant le système sont respectivement désignés par $V_{1}, V_{2}$ et $V_{3}, V_{1}$ étant le potentiel du cylindre d'entrée.

Ces trois grandeurs sont choisies de sorte que l'évolution du potentiel ait sur toute l'étendue du système un effet décélérateur. La valeur de $V_{2}$ est donc intermédiaire entre celles de $V_{1}$ et $V_{3}$ et $V_{3}$ est nécessairement de signe opposé à $V_{1}$ puisque l'on veut obtenir un rebroussement des particules.

Les deux paramètres électriques utilisés ici sont :

- le rapport des potentiels extrêmes $V_{3} / V_{1}$, qui est négatif,

- le rapport des différences de potentiel entre les cylindres, exprimé sous forme de pourcentage soit $R_{\mathrm{d}}=100 \frac{V_{1}-V_{2}}{V_{1}-V_{3}}$ dont la valeur est comprise entre 0 et 100 .

Pour $R_{\mathrm{d}}=0, V_{2}=V_{1}$, le miroir a le comportement d'un miroir bicylindrique.
Pour $R_{\mathrm{d}}=0, V_{2}=V_{3}$, le miroir a encore un comportement de miroir bicylindrique.

Pour $0<R_{\mathrm{d}}<100, V_{2} / V_{3}$ a une valeur comprise entre 1 et $V_{3} / V_{1}$, exprimée par :

$$
\frac{V_{2}}{V_{1}}=1-\frac{R_{\mathrm{d}}}{100}\left(1-\frac{V_{3}}{V_{1}}\right) .
$$

Une première étude a montré que pour des valeurs de $L$ allant jusqu'à $R$, les caractéristiques optiques du miroir en fonction de $V_{3} / V_{1}$ restent extrêmement voisines de celles du miroir bicylindrique en fonction de $V_{2} / V_{1}$ (Fig. 1) et ceci quelles que soient les valeurs pouvant être retenues pour les deux autres paramètres.

Pour une modification des propriétés, il faut d'une part un écartement suffisant entre les électrodes extrêmes et d'autre part créer une dissymétrie électrique convenable pour produire un étalement de la zone où se produit l'effet convergent du miroir.

Une valeur de l'écartement des cylindres extrêmes a semblé particulièrement intéressante :

$$
L=1,7 R ;
$$

elle correspond à la limite d'apparition de quatre zones dans le miroir (deux à effet divergent et deux à effet convergent). L'encombrement du système pour cette valeur de $L$ est encore raisonnable.

Ce premier paramètre étant choisi, deux géométries ont retenu notre attention; la première est désignée par «OMD», pour laquelle

$$
L_{\mathrm{e}}=0,9 R \text {, }
$$

la deuxième est désignée par " $O M E$ ", pour laquelle

$$
L_{\mathrm{e}}=1,5 \mathrm{R} \text {. }
$$

Pour chacune de ces géométries, nous étudions plusieurs répartitions entre les électrodes de la différence de potentiel globale. Dans la nomenclature utilisée ici, la dénomination de la géométrie est affectée d'un nombre représentant le pourcentage de la différence de potentiel globale, appliquée entre la première et la deuxième électrode.

Exemple : OMD 75 désigne un miroir de géométrie OMD, où $75 \%$ de la chute de potentiel totale sont appliqués entre la première et la deuxième électrode, la valeur de $R_{\mathrm{d}}$ correspondant à ce type de miroir est 75 .

3. Calcul des caractéristiques optiques. - 3.1 DéfINITIONS. - Le sens positif choisi sur l'axe $\mathbf{z}^{\prime} \mathbf{z}$ du système est le sens de propagation des particules incidentes; l'origine $O$ sur cet axe se trouve dans le plan de section droite terminant la première électrode (Fig. 2).

Les propriétés optiques du premier ordre sont entièrement déterminées par la connaissance de la position $\overline{\mathrm{OS}}$ du sommet du miroir équivalent et son rayon $\overline{\mathrm{SC}}$. Etant donné les conventions de signe le rayon $\overline{\mathrm{SC}}$ est négatif pour un miroir convergent et positif pour un miroir divergent. 
L'importance des défauts d'ouverture est chiffrée par la donnée du coefficient d'aberration sphérique principale $C_{\mathrm{s}_{0}}$. Si $\Delta l_{\mathrm{s}}=\overline{F_{\mathrm{g}}^{\prime} F_{\mathrm{a}}^{\prime}}$ représente l'aberration sphérique principale longitudinale pour un rayon se propageant à l'incidence parallèlement à l'axe à une distance $h$ de l'axe (Fig. 3), on a :

$$
\overline{\Delta l}_{\mathrm{s}}=C_{\mathrm{s}_{0}}\left(\frac{h}{f^{\prime}}\right)^{2}
$$

avec $f^{\prime}=\overline{\mathrm{SC}} / 2$ représentant la distance focale paraxiale.

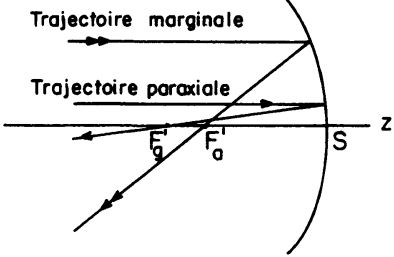

a) aberrations spheriques

$$
\Delta \ell_{s}=\overline{F_{g}^{\prime} F_{0}^{\prime}}
$$

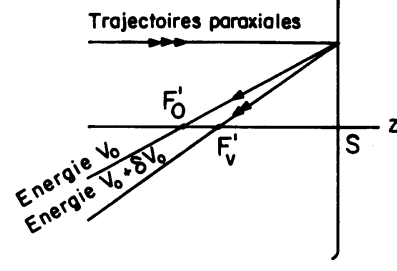

b) aberrotions chromatiques $\Delta \ell_{c}=\overline{F_{0}^{\prime} F_{v}^{\prime}}$
Fig. 3. - Définition des aberrations principales longitudinales.

[Definition of principal longitudinal aberrations.]

De même l'ordre de grandeur des défauts de chromatisme de position est obtenu par le calcul du coefficient $C_{\mathrm{c}}$ d'aberration chromatique principale. Si $\overline{\Delta l}_{\mathrm{c}}={\overline{F_{0}^{\prime} F_{\mathrm{v}}^{\prime}}}^{\prime}$ représente l'aberration chromatique principale longitudinale pour des rayons paraxiaux correspondant à des particules d'énergie cinétique $q V_{0}$ et $q\left(V_{0}+\delta V_{0}\right)$, le potentiel de la première électrode étant $-V_{0}$, on a :

$$
\overline{\Delta l}_{\mathrm{c}}=C_{\mathrm{c}} \frac{\delta V_{0}}{V_{0}}
$$

Ces diverses grandeurs sont déduites du calcul de trajectoires.

3.2 Calcul du potentiel et du Champ. - Dans ce calcul, les cylindres extrêmes sont supposés de longueur infinie; dans la réalité leur longueur doit être au moins égale à 2,5 fois leur rayon. Les espaces interélectrodes étant faibles $(0,1$ ou 0,4 fois le rayon des cylindres) le potentiel peut légitimement être approché aux limites de ces espaces par une fonction linéaire.

Les dérivées du potentiel en tout point de l'axe sont alors calculées par une méthode très simple que nous avons mise au point pour les systèmes à symétrie de révolution et rayon constant $[4,5]$.

Les composantes du champ en dehors de l'axe sont évaluées au moyen de développements limités à partir de leur valeur sur l'axe [6].

3.3 CalCUl Des TRAJeCtoires. - L'utilisation d'une méthode décrite par Weber [7] permet de connaître les trajectoires qu'elles soient paraxiales ou non. On en déduit alors les propriétés du premier et du troisième ordre.

A titre de vérification, nous avons aussi calculé les trajectoires paraxiales au moyen de la méthode dite de Recknagel [8].

Ces deux méthodes de conception fondamentalement différente donnent des résultats très voisins. $\mathrm{La}$ concordance est en général de l'ordre du $1000^{\mathrm{e}}$; l'écart devient toutefois un peu plus grand pour les miroirs faiblement convergents ou divergents, sans toutefois excéder $1 \%$.

De ce fait, il est raisonnable d'évaluer l'incertitude absolue sur la position du sommet à $0,05 R$. La précision sur le rayon est estimée à $1 \%$ et celle sur les coefficients d'aberration à environ $5 \%$

4. Résultats. - Les caractéristiques optiques du premier et du troisième ordre sont étudiées pour les deux types de miroirs d'une part en fonction du rapport $V_{3} / V_{1}$ des potentiels extrêmes et d'autre part en fonction de la distribution des tensions à l'intérieur du miroir.

Deux séries de quatre tableaux donnent :

- l'abscisse $\overline{\mathrm{OS}} \mathrm{du}$ sommet du miroir (Tableau I pour OMD et Tableau $\mathrm{V}$ pour OME),

- le rayon $\overline{\mathrm{SC}}$ du miroir (Tableaux II et IV),

\begin{tabular}{|c|c|c|c|c|c|c|c|c|c|c|}
\hline $\mathrm{V}_{1}^{\mathrm{TYPE}}$ & and 90 & an 80 & and 78 & AMD 76 & OMD 75 & OAD 74 & and 73 & $\operatorname{ard} 72$ & aD 71 & OND 70 \\
\hline$-0,10$ & 4,15 & 4,04 & 3,95 & 3,87 & 3,84 & 3,80 & 3,77 & 3,74 & 3,71 & 3,68 \\
\hline$-0,15$ & 2,73 & 3,14 & 3,13 & 3,11 & 3,10 & 3,08 & 3,07 & 3,06 & 3,04 & 3,03 \\
\hline$-0,20$ & 2,09 & 2,61 & 2,65 & 2,67 & 2,67 & 2,67 & 2,67 & 2,67 & 2,67 & $2,66$. \\
\hline$-0,25$ & 1,71 & 2,22 & 2,29 & 2,34 & 2,36 & 2,37 & 2,38 & 2,39 & 2,40 & 2,40 \\
\hline$-0,30$ & 1,47 & 1,92 & 2,00 & 2,07 & 2,09 & 2,12 & 2,14 & 2,16 & 2,18 & 2,19 \\
\hline$-0,35$ & 1,29 & 1,68 & 1,76 & 1,84 & 1,87 & 1,90 & 1,93 & 1,96 & 1,98 & 2,00 \\
\hline$-0,40$ & 1,15 & 1,49 & 1,57 & 1,64 & 1,68 & 1,71 & 1,75 & 1,78 & 1,81 & 1,83 \\
\hline$-0,45$ & 1,05 & 1,34 & 1,41 & 1,48 & 1,52 & 1,55 & 1,59 & 1,62 & 1,65 & 1,68 \\
\hline$-0,50$ & 0,96 & 1,22 & 1,29 & 1,35 & 1,38 & 1,42 & 1,45 & 1,48 & 1,51 & 1,54 \\
\hline$-0,55$ & 0,89 & 1,12 & 1,18 & 1,24 & 1,27 & 1,30 & 1,33 & 1,36 & 1,39 & 1,42 \\
\hline$-0,60$ & 0,82 & 1,03 & 1,09 & 1,14 & 1,17 & 1,20 & 1,23 & 1,26 & 1,29 & 1,32 \\
\hline$-0,65$ & 0,77 & 0,96 & 1,03 & 1,05 & 1,08 & 1,11 & 1,14 & 1,17 & 1,19 & 1,22 \\
\hline$-0,70$ & 0,72 & 0,89 & 0,94 & 0,99 & 1,02 & 1,04 & 1,05 & 1,07 & 1,09 & 1,13 \\
\hline$-0,75$ & 0,67 & 0,84 & 0,88 & 0,92 & 0,95 & 0,97 & 0,99 & 1,02 & 1,04 & 1,08 \\
\hline$-0,80$ & 0,63 & 0,79 & 0,83 & 0,87 & 0,89 & 0,91 & 0,93 & 0,95 & 0,98 & 1,00 \\
\hline$-0,85$ & 0,59 & 0,74 & 0,78 & 0,82 & 0,84 & 0,86 & 0,88 & 0,90 & 0,92 & 0,95 \\
\hline$-0,90$ & 0,56 & 0,70 & 0,74 & 0,77 & 0,79 & 0,81 & 0,83 & 0,85 & 0,87 & 0,89 \\
\hline$-0,95$ & 0,53 & 0,66 & 0,70 & 0,73 & 0,75 & 0,77 & 0,78 & 0,80 & 0,82 & 0,84 \\
\hline$-1,00$ & 0,50 & 0,63 & 0,66 & 0,69 & 0,71 & 0,73 & 0,74 & 0,76 & 0,78 & 0,80 \\
\hline
\end{tabular}

Tableau I. - Abscisse du sommet des miroirs OMD.

[Abscissa of the summit of the OMD mirrors.] 
Tableau II. - Rayon des miroirs OMD (- pour les miroirs convergents, + pour les miroirs divergents).

[Radius of curvature of the OMD mirrors ( - for convergent mirrors, + for divergent mirrors).]

\begin{tabular}{|c|c|c|c|c|c|c|c|c|c|c|}
\hline ThPE & avio 90 & and 80 & OND 78 & aMd 76 & OND 75 & OND 74 & and 73 & OND 72 & OMD 71 & OND 70 \\
\hline$-0,10$ & $-4,99$ & $-5,61$ & $-5,55$ & $-5,46$ & $-5,40$ & $-5,33$ & $-5,26$ & $-5,18$ & $-5,10$ & $-5,02$ \\
\hline$-0,15$ & $-3,82$ & $-5,81$ & $-6,21$ & $-6,56$ & $-6,71$ & $-6,84$ & $\mid-6,94$ & $-7,01$ & $-7,06$ & $-7,08$ \\
\hline$-0,20$ & $-3,46$ & $-5,64$ & $-6,45$ & $-7,44$ & $-8,01$ & $-8,61$ & $-9,26$ & $-9,94$ & $-1,0601$ & $-1,1401$ \\
\hline$-0,25$ & $-3,51$ & $-5,24$ & $-6,11$ & $-7,38$ & $-8,21$ & $-9,25$ & $-1,05$ o1 & $-1,2201$ & $\begin{array}{lll}-1,43 & 01\end{array}$ & $\mid \begin{array}{ll}-1,71 & 01\end{array}$ \\
\hline$-0,30$ & $-3,89$ & $-4,97$ & $-5,71$ & $-6,85$ & $-7,66$ & $-8,71$ & $\mid \begin{array}{ll}-1,01 & 01\end{array}$ & $\begin{array}{ll}-1,21 & 01\end{array}$ & $\begin{array}{lll}-1, & 5 & 01\end{array}$ & $-1,9601$ \\
\hline$-0,35$ & $-4,73$ & $-4,99$ & $-5,54$ & $-6,45$ & $-7,10$ & $-7,97$ & $-9,16$ & $\mid-1,0801$ & $\left|\begin{array}{lll}-1,34 & 01\end{array}\right|$ & $-1,7501$ \\
\hline$-0,40$ & $-6,47$ & $-5,36$ & $-5,73$ & $-6,40$ & $-6,90$ & $-7,58$ & $-8,50$ & $-9,79$ & $\begin{array}{lll}-1,17 & 01\end{array}$ & $-1,4701$ \\
\hline$-0,45$ & $\begin{array}{ll}-1,10 & 01\end{array}$ & $-6,24$ & $-6,37$ & $-6,81$ & $-7,17$ & $-7,69$ & $-8,40$ & $-9,39$ & $-1,0801$ & $-1,3001$ \\
\hline$-0,50$ & $\begin{array}{ll}-4,37 & 01\end{array}$ & $-8,09$ & $-7,78$ & $-7,88$ & $-8,10$ & $-8,46$ & $-8,99$ & $-9,77$ & $-1,0901$ & $-1,2601$ \\
\hline$-0,55$ & 2,1301 & $\begin{array}{ll}-1,26 & 01\end{array}$ & $-1,0901$ & $-1,0301$ & $|-1,0201|$ & $-1,0301$ & $-1,0601$ & $\begin{array}{lll}-1,12 & 01\end{array}$ & $\mid-1,2101$ & $\begin{array}{lll}-1,35 & 01\end{array}$ \\
\hline$-0,60$ & 8,53 & $\begin{array}{lll}-3,41 & 01\end{array}$ & $\left|\begin{array}{ll}-2,10 & 01\end{array}\right|$ & $-1,6501$ & $\begin{array}{lll}-1,54 & 01\end{array}$ & $\mid \begin{array}{lll}-1,48 & 01\end{array}$ & $-1,4601$ & $\left|\begin{array}{ll}-1,48 & 01\end{array}\right|$ & $\left|\begin{array}{ll}-1,54 & 01\end{array}\right|$ & $-1,6601$ \\
\hline$-0,65$ & 5,35 & 4,0901 & $\left|\begin{array}{ll}1,01 & 03\end{array}\right|$ & $\left|\begin{array}{lll}-5,59 & 01\end{array}\right|$ & $-3,9501$ & $-3,1901$ & $\begin{array}{lll}-2,80 & 01\end{array}$ & $\begin{array}{lll}-2,60 & 01\end{array}$ & $\begin{array}{lll}-2,53 & 01\end{array}$ & $-2,5801$ \\
\hline-0.70 & 3,91 & $\begin{array}{lll}1,24 & 01\end{array}$ & $\begin{array}{lll}1,89 & 01\end{array}$ & $\begin{array}{lll}3,44 & 01\end{array}$ & $\begin{array}{lll}5,28 & 01\end{array} \mid$ & 9,9701 & 3,9902 & $\left|\begin{array}{ll}-2,82 & 02\end{array}\right|$ & $-1,2602$ & $\begin{array}{lll}-9,43 & 01\end{array}$ \\
\hline$-0,75$ & 3,10 & 7,23 & 9,33 & 1,2601 & $\begin{array}{lll}1,50 & 01\end{array} \mid$ & $\begin{array}{lll}1,81 & 01\end{array}$ & 2,2201 & $\left|\begin{array}{ll}2,76 & 01\end{array}\right|$ & 3,4601 & $\begin{array}{lll}4,37 & 01\end{array}$ \\
\hline$-0,80$ & 2,58 & 5,09 & 6,15 & 7,62 & 8,57 & 9,69 & $\begin{array}{llll}1,10 & 01\end{array}$ & $1,2601 \mid$ & 1,4401 & 1,6501 \\
\hline$-0,85$ & 2,21 & 3,93 & 4,58 & 5,43 & 5,95 & 6,54 & 7,22 & 7,99 & 8,86 & 9,82 \\
\hline$-0,90$ & 1,95 & 3,20 & 3,65 & 4,21 & 4,54 & 4,91 & 5,39 & 5,80 & 6,31 & 6,88 \\
\hline$-0,95$ & 1,75 & 2,71 & 3,04 & 3,44 & 3,67 & 3,92 & 4,21 & 4,52 & 4,87 & 5,25 \\
\hline$-1,00$ & 1,59 & 2,36 & 2,61 & 2,90 & 3,08 & 3,27 & 3,47 & 3,70 & 3,95 & 4,23 \\
\hline
\end{tabular}

N.B. L'indication $0 n$ indique la puissance de 10 par laquelle il faut multiplier le nombre.

- le coefficient $C_{\mathrm{s}_{0}}$ d'aberration sphérique principale (Tableau III et Tableau VII),

- le coefficient $C_{\mathrm{c}}$ d'aberration chromatique principale (Tableau IV et Tableau VIII).

Ces quatre grandeurs ont la dimension d'une longueur. Les résultats sont donc exprimés avec l'unité de longueur choisie dans cette étude, à savoir le rayon des cylindres constituant le système.

Quelques remarques peuvent être faites sur les résultats obtenus.

1) Pour les deux géométries considérées et pour les rapports $R_{\mathrm{d}}$ étudiés, la position du sommet en fonction du rapport $V_{3} / V_{1}$ des potentiels extrêmes suit une évolution tout à fait semblable à celle observée pour le miroir bicylindrique dans la gamme correspondante des valeurs $V_{2} / V_{1}$ et représentée figure 1 .

2) Les figures 4 et 5 donnent les variations du rayon de courbure de quelques miroirs $\mathrm{OMD}$ et $\mathrm{OME}$ en fonction du rapport $V_{3} / V_{1}$. Les courbes obtenues pour les miroirs OMD 90 et OME 90 sont peu différentes de celle de la figure 1 représentant le rayon de courbure du miroir bicylindrique en fonction de $V_{2} / V_{1}$ et cela est tout à fait normal puisque la
Tableau III. - Coefficient d'aberration sphérique principale des miroirs $O M D$.

[Principal spherical aberration coefficient of the OMD mirrors.]

\begin{tabular}{|c|c|c|c|c|c|c|c|c|c|c|}
\hline T/N & OMO 90 & OMD 80 & OMD 78 & OMD 76 & OMO 75 & OMD 74 & OMD 73 & OMD 72 & OMD 71 & OMD 70 \\
\hline$-0,10$ & $\left|\begin{array}{ll}-1,8 & 01\end{array}\right|$ & $-1,8$ of & $\mid \begin{array}{ll}-2,0 & 01\end{array}$ & $\left|\begin{array}{ll}-2,0 & 01\end{array}\right|$ & $\left|\begin{array}{ll}-2,1 & 01\end{array}\right|$ & {$\left[\begin{array}{ll}-2,0 & 01\end{array}\right]$} & $\begin{array}{ll}-2,0 & 01\end{array}$ & $\begin{array}{ll}-1,9 & 01\end{array}$ & $\begin{array}{ll}-1,9 & 01\end{array}$ & $\begin{array}{ll}-1,8 & 01\end{array}$ \\
\hline$-0,15$ & 1,1 & $-1,3$ of & $\begin{array}{ll}-2,5 & 01\end{array}$ & $\mid \begin{array}{ll}-3,9 & 01\end{array}$ & $\begin{array}{ll}-4,7 & 01\end{array}$ & {$\left[\begin{array}{ll}-5,5 & 01\end{array}\right.$} & $-6,3$ ol & $\mid \begin{array}{ll}-7,0 & 01\end{array}$ & $-7,4$ l 01 & $\begin{array}{lll}-7,8 & 01\end{array}$ \\
\hline$-0,20$ & 1,7 & $\begin{array}{ll}-1,6 & 01\end{array}$ & $\mid \begin{array}{ll}-1,8 & 01\end{array}$ & $\mid \begin{array}{ll}-5,6 & 01\end{array}$ & $\begin{array}{ll}-9,2 & 01\end{array}$ & $\mid \begin{array}{ll}-1,5 & 02\end{array}$ & $\begin{array}{ll}-2,2 & 02\end{array}$ & $\begin{array}{ll}-3,2 & 02 \\
\end{array}$ & $\begin{array}{ll}-4.7 & 02\end{array}$ & $\begin{array}{ll}-6,3 & 02 \\
\end{array}$ \\
\hline$-0,25$ & 2,5 & $\begin{array}{ll}1,1 & 01\end{array}$ & 7,9 & $\left|\begin{array}{ll}-1,8 & 01\end{array}\right|$ & $\left|\begin{array}{ll}-5,3 & 01\end{array}\right|$ & $\mid \begin{array}{ll}-1,3 & 02\end{array}$ & $\begin{array}{ll}-2,9 & 02\end{array}$ & $\begin{array}{ll}-6,1 & 02\end{array}$ & $\begin{array}{ll}-1,4 & 03\end{array}$ & $-3,3 \quad 03$ \\
\hline$-0,30$ & 3,9 & $\begin{array}{ll}1,9 & 01\end{array}$ & $\left|\begin{array}{ll}2,5 & 01\end{array}\right|$ & $\begin{array}{ll}3,0 & 01\end{array}$ & $\begin{array}{ll}2.5 & 01\end{array}$ & 3,5 & $\begin{array}{ll}-6,6 & 01\end{array}$ & $\begin{array}{ll}-2,8 & 02\end{array}$ & $\begin{array}{ll}-1,1 & 03\end{array}$ & $\begin{array}{ll}-4,1 & 03\end{array}$ \\
\hline$-0,35$ & 9,2 & $\begin{array}{ll}2,7 & 01\end{array}$ & $\mid \begin{array}{ll}3.9 & 01\end{array}$ & $\begin{array}{ll}5,8 & 01\end{array}$ & $\mid \begin{array}{ll}7.5 & 01\end{array}$ & $\begin{array}{ll}9,2 & 01\end{array}$ & $1,1 \quad 02$ & $1,2 \quad 02$ & $\begin{array}{ll}6,7 & 01\end{array}$ & $\begin{array}{ll}-6,3 & 03\end{array}$ \\
\hline$-0,40$ & $\begin{array}{ll}2,9 & 01\end{array}$ & $\begin{array}{ll}4,3 & 01\end{array}$ & $\mid \begin{array}{ll}5,7 & 01\end{array}$ & $\begin{array}{ll}8,3 & 01\end{array}$ & $\begin{array}{ll}1,1 & 02\end{array}$ & $\begin{array}{ll}1,4 & 02\end{array}$ & 2,002 & $\begin{array}{ll}3,0 & 02\end{array}$ & $\begin{array}{ll}4,7 & 02\end{array}$ & $\begin{array}{ll}8,1 & 02\end{array}$ \\
\hline$-0,45$ & $\begin{array}{ll}2.0 & 02\end{array}$ & $\begin{array}{ll}8,1 & 01\end{array}$ & $\left|\begin{array}{ll}9.9 & 01\end{array}\right|$ & $\mid \begin{array}{ll}1,3 & 02\end{array}$ & $\left|\begin{array}{ll}1,6 & 02\end{array}\right|$ & $\begin{array}{ll}2,1 & 02\end{array}$ & $\begin{array}{ll}2,8 & 02\end{array}$ & 4,1 02 & $\begin{array}{ll}6,5 & 02\end{array}$ & $\begin{array}{ll}1.2 & 03\end{array}$ \\
\hline$-0,50$ & $\begin{array}{ll}3,1 & 04\end{array}$ & 2,502 & $\begin{array}{ll}2,4 & 02\end{array}$ & $\begin{array}{ll}2,6 & 02\end{array}$ & $\begin{array}{ll}3.0 & 02\end{array}$ & $\begin{array}{ll}3,5 & 02\end{array}$ & $\begin{array}{ll}4,5 & 02\end{array}$ & $\begin{array}{ll}6,4 & 02\end{array}$ & $\begin{array}{ll}9.3 & 02\end{array}$ & $\begin{array}{lll}1,5 & 03\end{array}$ \\
\hline$-0,55$ & $\mid \begin{array}{ll}-4,1 & 02\end{array}$ & $\begin{array}{ll}1,4 & 03\end{array}$ & $\begin{array}{ll}8.7 & 02\end{array}$ & $\left|\begin{array}{ll}7,7 & 02\end{array}\right|$ & $\begin{array}{ll}8,2 & 02\end{array}$ & $\begin{array}{ll}8.8 & 02\end{array}$ & $\begin{array}{ll}1,0 & 03\end{array}$ & 1,203 & $\begin{array}{ll}1,6 & 03\end{array}$ & $\begin{array}{lll}2.6 & 03\end{array}$ \\
\hline$-0,60$ & $\begin{array}{ll}-5,5 & 01\end{array}$ & $\begin{array}{lll}6,8 & 04\end{array}$ & $\begin{array}{ll}1,2 & 04\end{array}$ & {$\left[\begin{array}{ll}5,1 & 03\end{array}\right]$} & $\mid \begin{array}{ll}4,2 & 03\end{array}$ & $\begin{array}{ll}3,8 & 03\end{array}$ & $\begin{array}{ll}3.7 & 03\end{array}$ & $\begin{array}{lll}4,0 & 03\end{array}$ & $\left|\begin{array}{ll}4,9 & 03\end{array}\right|$ & $\begin{array}{lll}6,6 & 03\end{array}$ \\
\hline$-0,65$ & $\begin{array}{ll}-2,5 & 01\end{array}$ & $\begin{array}{ll}1,1 & 05\end{array}$ & $\begin{array}{ll}1,3 & 11\end{array}$ & $\begin{array}{ll}7,0 & 05\end{array}$ & $\begin{array}{ll}1,8 & 05\end{array}$ & $\begin{array}{ll}7.8 & 04\end{array}$ & $\begin{array}{lll}5,0 & 04\end{array}$ & $\begin{array}{ll}4,2 & 04\end{array}$ & $\begin{array}{ll}3.7 & 04\end{array}$ & $\begin{array}{ll}4,0 & 04\end{array}$ \\
\hline$-0,70$ & $\begin{array}{ll}-1,1 & 01\end{array}$ & $8,7 \quad 02$ & $\begin{array}{ll}6,6 & 03\end{array}$ & $\begin{array}{ll}8,3 & 04\end{array}$ & $\begin{array}{ll}5,3 & 05\end{array}$ & $\begin{array}{ll}9,0 & 07\end{array}$ & $\begin{array}{ll}8,0 & 08\end{array}$ & $\begin{array}{lll}4,2 & 08\end{array}$ & $\begin{array}{ll}2,5 & 07\end{array}$ & $\begin{array}{ll}7,1 & 06\end{array}$ \\
\hline$-0,75$ & $-6,4$ & $\begin{array}{ll}6.5 & 01\end{array}$ & \begin{tabular}{|ll}
3,4 & 02
\end{tabular} \mid & $\begin{array}{ll}1.4 & 03\end{array}$ & $\left|\begin{array}{ll}3,2 & 03\end{array}\right|$ & $\begin{array}{ll}7,6 & 03\end{array} \mid$ & 1,904 & 4,604 & $\begin{array}{ll}1.4 & 05\end{array}$ & $\begin{array}{lll}3,7 & 05\end{array}$ \\
\hline$-0,80$ - & $-4,4$ & $\begin{array}{ll}1,1 & 01\end{array}$ & $\begin{array}{ll}4,6 & 01\end{array}$ & $\begin{array}{ll}1,7 & 02\end{array}$ & $\begin{array}{ll}2,9 & 02\end{array} \mid$ & $\begin{array}{ll}5,8 & 02\end{array}$ & $\begin{array}{ll}9,9 & 02\end{array}$ & $\begin{array}{lll}2,0 & 03\end{array}$ & $\begin{array}{ll}3,5 & 03\end{array}$ & $\begin{array}{ll}6,8 & 04\end{array}$ \\
\hline$-0,85$ & $-3,0$ & $-4,1 \quad 01$ & 2,7 & $\begin{array}{ll}3,0 & 01\end{array}$ & $\mid \begin{array}{ll}6,2 & 01\end{array}$ & $\begin{array}{ll}8,5 & 01\end{array}$ & $\begin{array}{ll}1,7 & 02\end{array}$ & 2,602 & 4,402 & $\begin{array}{lll}7,9 & 02\end{array}$ \\
\hline$-0,90$ - & $-2,2$ & $-1,9$ & $\begin{array}{lll}-6,0 & -01\end{array}$ & 5,1 & $\begin{array}{ll}1,5 & 01\end{array}$ & $\begin{array}{ll}1,9 & 01\end{array}$ & $\begin{array}{lll}4,0 & 01\end{array}$ & $\begin{array}{ll}5,8 & 01\end{array}$ & $\begin{array}{ll}1,1 & 02\end{array}$ & $\begin{array}{ll}1,6 & 02\end{array}$ \\
\hline$-0,95$. & $-1,6$ & $-1,9$ & $-2,0$ & $\left|\begin{array}{ll}-5,2 & -01\end{array}\right|$ & 2,9 & 3,9 & $\begin{array}{ll}1,2 & 01\end{array}$ & $\begin{array}{ll}1,6 & 01\end{array}$ & $\begin{array}{ll}2,9 & 01\end{array}$ & $\begin{array}{lll}4,5 & 01\end{array}$ \\
\hline$-1,00$. & $-1,4$ & $-2,0$ & $-1,7$ & $-1,5$ & $\begin{array}{lll}-2,2 & -01\end{array}$ & $\left|\begin{array}{ll}-1,8 & -01\end{array}\right|$ & 3,5 & 4,4 & 9,5 & 1,801 \\
\hline
\end{tabular}

Tableau IV. - Coefficient d'aberration chromatique principale des miroirs $O M D$.

[Principal chromatic aberration coefficient of the OMD mirrors.]

\begin{tabular}{|c|c|c|c|c|c|c|c|c|c|c|}
\hline 3$)^{Y P E}$ & OMO 90 & OMD 80 & OMO 78 & OMD 76 & OMO 75 & OMD 74 & OMD 73 & OMO 72 & OMO 71 & OMD 70 \\
\hline$-0,10$ & $\begin{array}{ll}2.7 & 01\end{array}$ & $3,1 \quad 01$ & $3,1 \quad 01$ & $\left.\mid \begin{array}{ll}3,3 & 01\end{array}\right]$ & $\left|\begin{array}{ll}3,2 & 01\end{array}\right|$ & $\left|\begin{array}{ll}3.5 & 01\end{array}\right|$ & $\left|\begin{array}{ll}3,4 & 01\end{array}\right|$ & $\left|\begin{array}{ll}3,4 & 01\end{array}\right|$ & $\left|\begin{array}{ll}3,3 & 01\end{array}\right|$ & 3,3 o1 \\
\hline$-0,15$ & $\begin{array}{ll}1,3 & 01\end{array}$ & $\begin{array}{ll}1,6 & 01\end{array}$ & 2,001 & $\begin{array}{ll}2,7 & 01\end{array}$ & $\begin{array}{ll}3,1 & 01\end{array}$ & $\mid \begin{array}{ll}3,3 & 01\end{array}$ & $\left|\begin{array}{ll}3,8 & 01\end{array}\right|$ & $\begin{array}{ll}3,9 & 01\end{array}$ & $\begin{array}{ll}4,2 & 01\end{array}$ & $\begin{array}{ll}4,6 & 01\end{array}$ \\
\hline$-0,20$ & 0,9 & 6,2 & 8,4 & $1,4 \quad 01$ & $\left|\begin{array}{ll}1,8 & 01\end{array}\right|$ & $\left.\begin{array}{ll}2,5 & 01\end{array}\right]$ & $\begin{array}{ll}3,3 & 01\end{array}$ & $\left|\begin{array}{ll}4,3 & 01\end{array}\right|$ & $\begin{array}{ll}5,8 & 01\end{array}$ & $\begin{array}{ll}7,4 & 01\end{array}$ \\
\hline$-0,25$ & $\begin{array}{ll}1,0 & 01\end{array}$ & 3,7 & 2,5 & 2,1 & 3,1 & 5,6 & $\begin{array}{ll}1,1 & 01\end{array}$ & $\begin{array}{ll}2,0 & 01\end{array}$ & $\begin{array}{ll}3,8 & 01\end{array}$ & $\begin{array}{ll}6.9 & 01\end{array}$ \\
\hline$-0,30$ & $\begin{array}{ll}1,3 & 01\end{array}$ & 5,2 & 2,7 & $-3,8-01$ & $-2,2$ & 4,1 & $-6,1$ & $-7,3$ & $-6,8$ & $4,9-03$ \\
\hline$-0,35$ & $\begin{array}{ll}2,0 & 01\end{array}$ & 8,2 & 5,8 & 2,3 & $\left|\begin{array}{ll}1,1 & -01\end{array}\right|$ & 2,9 & $-6,9$ & $\begin{array}{ll}-1,2 & 01\end{array}$ & $\begin{array}{ll}-2,1 & 01\end{array}$ & $\begin{array}{ll}-3,6 & 01\end{array}$ \\
\hline$-0,40$ & $\begin{array}{ll}3,9 & 01\end{array}$ & $\begin{array}{ll}1,3 & 01\end{array}$ & $\begin{array}{ll}1,0 & 01\end{array}$ & 7,4 & 5,2 & 2,7 & $\begin{array}{lll}-7,7 & -01\end{array}$ & $-5,9$ & $\begin{array}{ll}-1,3 & 01\end{array} \mid$ & $\begin{array}{ll}-2,8 & 01\end{array}$ \\
\hline$-0,45$ & $1,2 \quad 02$ & $2,2 \quad 01$ & $\begin{array}{ll}1.8 & 01\end{array}$ & $\begin{array}{ll}1,4 & 01\end{array}$ & $\begin{array}{ll}1,3 & 01\end{array}$ & $\begin{array}{ll}1.0 & 01\end{array}$ & 7,6 & 3,7 & $-1,7$ & $-1,1 \quad 01$ \\
\hline-0.50 & $\begin{array}{ll}2.0 & 03\end{array}$ & 4,4 o1 & $\begin{array}{ll}3,4 & 01\end{array}$ & $\begin{array}{ll}2,7 & 01\end{array}$ & $\left|\begin{array}{ll}2,4 & 01\end{array}\right|$ & $\left|\begin{array}{ll}2,2 & 01\end{array}\right|$ & $\begin{array}{ll}1,9 & 01\end{array}$ & $\left|\begin{array}{ll}1,6 & 01\end{array}\right|$ & $\begin{array}{ll}1,3 & 01\end{array} \mid$ & 6,9 \\
\hline$-0,55$ & $\begin{array}{ll}5,0 & 02\end{array}$ & $1,2 \quad 02$ & $\begin{array}{ll}7.6 & 01\end{array}$ & $\begin{array}{ll}5,7 & 01\end{array}$ & $\begin{array}{ll}5,0 & 01\end{array}$ & $\left|\begin{array}{ll}4,5 & 01\end{array}\right|$ & $\left|\begin{array}{ll}4,1 & 01\end{array}\right|$ & $\begin{array}{ll}3,8 & 01\end{array}$ & $\begin{array}{ll}3,4 & 01\end{array}$ & $\begin{array}{ll}3,1 & 01\end{array}$ \\
\hline$-0,60$ & $8,1 \quad 01$ & $\begin{array}{ll}9,7 & 02\end{array}$ & $\begin{array}{ll}3,3 & 02\end{array}$ & $\begin{array}{ll}1,7 & 02\end{array}$ & $\begin{array}{ll}1,5 & 02\end{array}$ & $\left|\begin{array}{ll}1,2 & 02\end{array}\right|$ & $\begin{array}{ll}1,0 & 02\end{array}$ & $\begin{array}{ll}9,6 & 01\end{array}$ & $\left|\begin{array}{ll}8.5 & 01\end{array}\right|$ & $8,1 \quad 01$ \\
\hline$-0,65$ & $\begin{array}{ll}3,5 & 01\end{array}$ & $\begin{array}{ll}1.5 & 03\end{array}$ & $\begin{array}{ll}8,6 & 05\end{array}$ & $\begin{array}{ll}2,3 & 03\end{array}$ & $\left|\begin{array}{ll}1,1 & 03\end{array}\right|$ & $\mid \begin{array}{ll}6,7 & 02\end{array}$ & $\begin{array}{ll}4,8 & 02\end{array}$ & $\begin{array}{ll}3,6 & 02\end{array}$ & $\begin{array}{ll}3,0 & 02\end{array}$ & $2,8 \quad 02$ \\
\hline$-0,70$ & 2,001 & $\begin{array}{ll}1,5 & 02\end{array}$ & $\begin{array}{ll}3,3 & 02\end{array}$ & $\begin{array}{ll}1,0 & 03\end{array}$ & $\begin{array}{ll}2,2 & 03\end{array}$ & $\left|\begin{array}{ll}7,5 & 03\end{array}\right|$ & $\begin{array}{ll}1,1 & 05\end{array}$ & 5,104 & $\mid \begin{array}{ll}7,5 & 03\end{array}$ & $\begin{array}{ll}4,7 & 03\end{array}$ \\
\hline$-0,75$ & $\begin{array}{ll}1,3 & 01\end{array}$ & $\begin{array}{ll}5,4 & 01\end{array}$ & $8,9 \quad 01$ & $\begin{array}{ll}1,4 & 02\end{array}$ & $\begin{array}{ll}2,0 & 02\end{array} \mid$ & $\left|\begin{array}{ll}2,8 & 02\end{array}\right|$ & \begin{tabular}{|ll}
3,8 & 02
\end{tabular} \mid & $\begin{array}{ll}5,7 & 02\end{array}$ & $8,1 \quad 02$ & $\begin{array}{ll}1,2 & 03\end{array}$ \\
\hline$-0,80$ & 9,3 & $\begin{array}{ll}2,8 & 01\end{array}$ & $\begin{array}{ll}4,0 & 01\end{array}$ & $\begin{array}{ll}5,3 & 01\end{array}$ & $\begin{array}{ll}6,9 & 01\end{array}$ & $8,2 \quad 01$ & $\begin{array}{ll}1,0 & 02\end{array}$ & $\left|\begin{array}{ll}1,3 & 02\end{array}\right|$ & $\begin{array}{ll}1,6 & 02\end{array}$ & 2.002 \\
\hline$-0,85$ & 7,2 & $\begin{array}{ll}1,8 & 01\end{array}$ & $2,3 \quad 01$ & $\begin{array}{ll}3,0 & 01\end{array}$ & $\begin{array}{lll}3.6 & 01\end{array}$ & $\begin{array}{ll}4,3 & 01\end{array} \mid$ & $\left(\begin{array}{ll}5,1 & 01\end{array}\right.$ & $\left|\begin{array}{ll}5,6 & 01\end{array}\right|$ & $\begin{array}{ll}6,6 & 01\end{array}$ & $\begin{array}{ll}7,9 & 01\end{array}$ \\
\hline$-0,90$ & 5,8 & $\begin{array}{ll}1,3 & 01\end{array}$ & $\begin{array}{ll}1,6 & 01\end{array}$ & $\begin{array}{ll}2,0 & 01\end{array}$ & $\left|\begin{array}{ll}2,2 & 01\end{array}\right|$ & $\begin{array}{ll}2,5 & 01\end{array} \mid$ & $\left|\begin{array}{ll}2,7 & 01\end{array}\right|$ & $\begin{array}{ll}3,2 & 01\end{array}$ & $\begin{array}{ll}3,5 & 01\end{array}$ & $\left|\begin{array}{ll}4,1 & 01\end{array}\right|$ \\
\hline$-0,95$ & 4,7 & 9,6 & $\begin{array}{ll}1,1 & 01\end{array}$ & $\begin{array}{ll}1,4 & 01\end{array}$ & $\begin{array}{ll}1,5 & 01\end{array}$ & $\begin{array}{ll}1,7 & 01\end{array}$ & $\begin{array}{ll}1,8 & 01\end{array} \mid$ & $\begin{array}{ll}2,1 & 01\end{array}$ & $\begin{array}{ll}2,3 & 01\end{array}$ & $\left|\begin{array}{ll}2,6 & 01\end{array}\right|$ \\
\hline$-1,00$ & 4,1 & 7,6 & 8,6 & $\begin{array}{ll}1,1 & 01\end{array}$ & $\begin{array}{ll}1,2 & 01\end{array}$ & $\begin{array}{ll}1,3 & 01\end{array}$ & $\begin{array}{ll}1,3 & 01\end{array}$ & $\begin{array}{ll}1,5 & 01\end{array}$ & $\begin{array}{ll}1,7 & 01\end{array}$ & $\begin{array}{ll}1,9 & 01\end{array}$ \\
\hline
\end{tabular}


Tableau V. - Abscisse du sommet des miroirs OME. [Abscissa of the summit of the OME mirrors.]

\begin{tabular}{|c|c|c|c|c|c|c|c|}
\hline$V_{3} x_{1}^{T Y P E}$ & OME 90 & ONE 85 & OME 80 & OME 75 & OME 70 & asE 65 & OE 60 \\
\hline$-0,15$ & 3,39 & 4,22 & 4,02 & 3,66 & 3,40 & 3,23 & 3,12 \\
\hline$-0,20$ & 2,25 & 3,03 & 3,40 & 3,29 & 3.08 & 2,96 & 2,85 \\
\hline$-0,25$ & 1,71 & 2,24 & 2,80 & 2,96 & 2,88 & 2,76 & 2,67 \\
\hline$-0,30$ & 1,39 & 1,76 & 2,25 & 2,62 & 2,67 & 2,61 & 2,53 \\
\hline$-0,35$ & 1,18 & 1,46 & 1,84 & 2,26 & 2,47 & 2,47 & 2,42 \\
\hline$-0,40$ & 1,03 & 1,25 & 1,55 & 1,93 & 2,23 & 2,34 & 2,32 \\
\hline$-0,45$ & 0,91 & 1,09 & 1,33 & 1,65 & 1,99 & 2,17 & 2,22 \\
\hline$-0,50$ & 0,82 & 0,97 & 1,17 & 1,44 & 1,76 & 2,02 & 2,12 \\
\hline$-0,55$ & 0,73 & 0,87 & 1,04 & 1,27 & 1,56 & 1,84 & 2.01 \\
\hline$-0,60$ & 0,68 & 0,79 & 0,94 & 1,13 & 1,39 & 1,67 & 1,91 \\
\hline$-0,65$ & 0,62 & 0,73 & 0,85 & 1,02 & 1,24 & 1,51 & 1,76 \\
\hline$-0,70$ & 0,57 & 0,66 & 0,75 & 0,93 & 1,12 & 1,37 & 1,63 \\
\hline$-0,75$ & 0,53 & 0,61 & 0,72 & 0,85 & 1,02 & 1,24 & 1,50 \\
\hline$-0,80$ & 0,48 & 0,57 & 0,66 & 0,78 & 0,94 & 1,14 & 1,39 \\
\hline$-0,85$ & 0,45 & 0,52 & 0,61 & 0,73 & 0,86 & 1,05 & 1,28 \\
\hline$-0,90$ & 0,41 & 0,48 & 0,57 & 0,67 & 0,79 & 0,97 & 1,18 \\
\hline$-0,95$ & 0,38 & 0,45 & 0,53 & 0,62 & 0,74 & 0,89 & 1,09 \\
\hline$-1,00$ & 0,35 & 0,41 & 0,49 & 0,58 & 0,70 & 0,82 & 1,02 \\
\hline
\end{tabular}

Tableau VI. - Rayon des miroirs OME (- pour les miroirs convergents, + pour les miroirs divergents).

[Radius of curvature of the OME mirrors.]

\begin{tabular}{|c|c|c|c|c|c|c|c|}
\hline TIXP & ane 90 & QNE 85 & aE 80 & aNE 75 & OEE 70 & aE 65 & aE 60 \\
\hline$-0,15$ & $-4,33$ & $-6,26$ & $-8,84$ & $\begin{array}{lll}-1,23 & 01\end{array}$ & $\left|\begin{array}{ll}-1,38 & 01\end{array}\right|$ & $\mid \begin{array}{ll}-1,18 & 01\end{array}$ & $-9,10$ \\
\hline$-0,20$ & $-3,32$ & $-4,52$ & $-7,10$ & $\begin{array}{lll}-1,54 & 01\end{array}$ & $\begin{array}{lll}-1,05 & 02\end{array} \mid$ & $\left|\begin{array}{lll}7,55 & 01\end{array}\right|$ & 1,2202 \\
\hline$-0,25$ & $-3,11$ & $-3,64$ & $-5,26$ & $\begin{array}{ll}-1,11 & 01\end{array} \mid$ & $\begin{array}{lll}1,24 & 02\end{array}$ & $\mid \begin{array}{lll}1,31 & 01\end{array}$ & 9,45 \\
\hline$-0,30$ & $-3,27$ & $-3,35$ & $-4,21$ & $-7,29$ & $|-6,91 \quad 01|$ & $\begin{array}{lll}1,02 & 01\end{array}$ & 5,89 \\
\hline$-0,35$ & $-3,77$ & $-3,42$ & $-3,75$ & $-5,40$ & $\left|\begin{array}{lll}-1,66 & 01\end{array}\right|$ & $\begin{array}{lll}1,22 & 01\end{array} \mid$ & 5,07 \\
\hline$-0,40$ & $-4,78$ & $-3,79$ & $-3,67$ & $-4,51$ & $-8,96$ & 2,4301 & 5,20 \\
\hline$-0,45 \mid$ & $-6,96$ & $-4,57$ & $-3,89$ & $-4,17$ & $-6,40$ & $\left|\begin{array}{lll}-8,81 & 01\end{array}\right|$ & 6,18 \\
\hline$-0,50$ & $-1,36$ or & $-6,11$ & $-4,43$ & $-4,17$ & $-5,32$ & $\begin{array}{lll}-1,57 & 01\end{array}$ & 8,69 \\
\hline$-0,55$ & $\begin{array}{lll}7,35 & 03\end{array}$ & $-9,85$ & $-5,50$ & $-4,48$ & $-4,91$ & $-9,27$ & 1,6401 \\
\hline$-0,60$ & 1,3501 & $-2,81 \quad 01$ & $-7,70$ & $-5,17$ & $-4,90$ & $-7,15$ & 1,2802 \\
\hline$-0,65$ & 6,74 & 3,0601 & $-1,3801$ & $-6,51$ & $-5,25$ & $-6,31$ & $\begin{array}{lll}-2,60 & 01\end{array}$ \\
\hline$-0,70$ & 4,52 & 9,83 & $-8,53$ o1 & $-9,35$ & $-6,02$ & $-6,11$ & $\begin{array}{ll}-1,34 & 01\end{array}$ \\
\hline$-0,75$ & 3,41 & 5,87 & 1,9601 & $\left|\begin{array}{lll}-1,81 & 01\end{array}\right|$ & $-7,53$ & $-6,37$ & $\begin{array}{lll}-1, & 0 & 01\end{array}$ \\
\hline$-0,80$ & 2,76 & 4,19 & 8,71 & $\mid \begin{array}{lll}-4,33 & 01\end{array}$ & $-1,08$ o1 & $-7,23$ & $-8,84$ \\
\hline$-0,85$ & 2,32 & 3,28 & 5,61 & $\begin{array}{lll}1,76 & 01\end{array}$ & $\begin{array}{lll}-2,00 & 01\end{array}$ & $-8,69$ & $-8,63$ \\
\hline$-0,90$ & 2,01 & 2,70 & 4,14 & 8,70 & $\begin{array}{lll}1,29 & 03\end{array}$ & $\left.\begin{array}{lll}-1, & 2 & 0\end{array}\right]$ & $-9,17$ \\
\hline$-0,95$ & 1,79 & 2,30 & 3,29 & 5,76 & 1,9401 & $\mid \begin{array}{lll}-2,16 & 01\end{array}$ & $-1,0601$ \\
\hline$-1,00$ & 1,61 & 2,02 & 2,74 & 4,31 & 9,63 & $-1,91 \quad 02$ & $-1,3801$ \\
\hline
\end{tabular}

Tableau VII. - Coefficient daberration sphérique principale des miroirs $O M E$.

[Principal spherical aberration coefficient of the OME mirrors.]

\begin{tabular}{|c|c|c|c|c|c|c|c|}
\hline$v_{3} /$ & OME 90 & OME $\quad 85$ & OME $\quad 80$ & OME 75 & OME 70 & OME 65 & OME 60 \\
\hline$-0,15$ & 3.7 & $\begin{array}{ll}3,0 & 01\end{array}$ & $-\begin{array}{ll}-1,5 & 01\end{array}$ & $\begin{array}{ll}-4,5 & 02\end{array}$ & $\begin{array}{ll}-1,0 & 03\end{array}$ & $\mid \begin{array}{ll}-5,3 & 02\end{array}$ & $\begin{array}{ll}-2,0 & 02\end{array}$ \\
\hline$-0,20$ & $\begin{array}{|cc|}6,6 & -01\end{array}$ & $\left|\begin{array}{ll}1,2 & 01\end{array}\right|$ & $\mid \begin{array}{ll}3,9 & 01\end{array}$ & $\begin{array}{ll}-1,1 & 03\end{array}$ & $-5,1 \quad 06$ & $-1,4$ 06 & $\begin{array}{ll}-7,3 & 06\end{array}$ \\
\hline$-0,25$ & $\begin{array}{lll}4.7 & -01\end{array}$ & 5,5 & $\begin{array}{ll}2,6 & 01\end{array}$ & $\begin{array}{ll}-6,7 & 01\end{array}$ & $-8,1 \quad 06$ & $\begin{array}{ll}-1,8 & 03\end{array}$ & $-6,1 \quad 02$ \\
\hline$-0,30$ & $7,0-01$ & 3,9 & $\begin{array}{ll}1,4 & 01\end{array}$ & $\begin{array}{ll}6,2 & 01\end{array}$ & $\begin{array}{ll}-4,8 & 05\end{array}$ & $\begin{array}{lll}-5,6 & 02\end{array}$ & $\begin{array}{ll}-1,0 & 02\end{array}$ \\
\hline$-0,35$ & 1,5 & 3,8 & 9,2 & 3,801 & $\begin{array}{ll}5,8 & 02\end{array} \mid$ & $\begin{array}{ll}-7,9 & 02\end{array}$ & $-4,1 \quad 01$ \\
\hline$-0,40$ & 2,9 & 5,1 & 8,6 & 2,501 & $\begin{array}{ll}1,8 & 02\end{array}$ & $\begin{array}{ll}-5,3 & 03\end{array}$ & $-3,4$ o1 \\
\hline$-0,45$ & $\begin{array}{ll}1,6 & 01\end{array}$ & $\left|\begin{array}{ll}1,0 & 01\end{array}\right|$ & 9,9 & $\begin{array}{ll}1,9 & 01\end{array}$ & $\left|\begin{array}{ll}9,0 & 01\end{array}\right|$ & $\begin{array}{lll}4,4 & 05\end{array}$ & $\begin{array}{ll}-3,7 & 01\end{array}$ \\
\hline$-0,50$ & $\begin{array}{ll}6,1 & 01\end{array}$ & $\left|\begin{array}{ll}2,7 & 01\end{array}\right|$ & $\left|\begin{array}{ll}1,6 & 01\end{array}\right|$ & $\begin{array}{ll}1,9 & 01\end{array}$ & $\left|\begin{array}{ll}5,6 & 01\end{array}\right|$ & $2,0 \quad 03$ & $-3,3$ o1 \\
\hline$-0,55$ & $\left|\begin{array}{ll}-3,7 & 12\end{array}\right|$ & $\begin{array}{ll}1,4 & 02\end{array}$ & $\left|\begin{array}{ll}3,3 & 01\end{array}\right|$ & $\mid \begin{array}{ll}2,5 & 01\end{array}$ & $\begin{array}{lll}4,4 & 01\end{array}$ & 4,202 & $\begin{array}{lll}9,8 & 02\end{array}$ \\
\hline$-0,60$ & $\left|\begin{array}{ll}-5,5 & 02\end{array}\right|$ & $\left|\begin{array}{ll}5,8 & 03\end{array}\right|$ & $\begin{array}{ll}1,2 & 02\end{array}$ & $\begin{array}{ll}4,1 & 01\end{array}$ & $\mid \begin{array}{ll}4,4 & 01\end{array}$ & $\begin{array}{ll}2,0 & 02\end{array}$ & $8,3 \quad 06$ \\
\hline$-0,65$ & $\left|\begin{array}{ll}-5,4 & 01\end{array}\right|$ & $\left|\begin{array}{ll}-9,2 & 02\end{array}\right|$ & $\begin{array}{ll}1,0 & 03\end{array} \mid$ & $\left|\begin{array}{ll}9,4 & 01\end{array}\right|$ & $\mid \begin{array}{ll}5,8 & 01\end{array}$ & $\begin{array}{ll}1,4 & 02\end{array}$ & $\begin{array}{lll}-2,7 & 04\end{array}$ \\
\hline$-0,70$ & $\left|\begin{array}{ll}-2,1 & 01\end{array}\right|$ & $\left|\begin{array}{ll}-6,4 & 01\end{array}\right|$ & $\begin{array}{ll}1,1 & 06\end{array}$ & $\begin{array}{ll}3,8 & 02\end{array}$ & $\begin{array}{ll}9,6 & 01\end{array}$ & $\begin{array}{ll}1,3 & 02\end{array}$ & $\begin{array}{ll}-2,5 & 03\end{array}$ \\
\hline$-0,75$ & $-9,9$ & $\left|\begin{array}{ll}-2,9 & 01\end{array}\right|$ & $\begin{array}{ll}2,1 & 03\end{array}$ & $\begin{array}{ll}4,6 & 03\end{array}$ & \begin{tabular}{|ll}
2,6 & 02
\end{tabular} & $\begin{array}{ll}1,5 & 02\end{array}$ & $\begin{array}{ll}9,5 & 02\end{array}$ \\
\hline$-0,80$ & $-5,7$ & $\left|\begin{array}{ll}-1,4 & 01\end{array}\right|$ & $\begin{array}{ll}4,1 & 01\end{array}$ & $\begin{array}{ll}4,3 & 10\end{array}$ & $\mid \begin{array}{ll}9,2 & 02\end{array}$ & $\begin{array}{ll}2,4 & 02\end{array}$ & $\begin{array}{ll}6,6 & 02\end{array}$ \\
\hline$-0,85$ & $-3,7$ & $-7,7$ & $\begin{array}{ll}-1,7 & 01\end{array}$ & $\begin{array}{ll}2,1 & 03\end{array}$ & \begin{tabular}{|ll}
1,1 & 04
\end{tabular} & $\begin{array}{ll}5,2 & 02\end{array}$ & $\begin{array}{ll}6,0 & 02\end{array}$ \\
\hline$-0,90$ & $-2,6$ & $-4,9$ & $\begin{array}{ll}-1,0 & 01\end{array}$ & $\begin{array}{lll}6,5 & 01\end{array}$ & $2,1 \quad 11$ & $\begin{array}{ll}1,8 & 03\end{array}$ & $\begin{array}{ll}8,3 & 02\end{array}$ \\
\hline$-0,95$ & $-2,0$ & $-3,1$ & $\mid-6,6$ & $-5,8$ & $\begin{array}{ll}6,6 & 03\end{array}$ & $\begin{array}{ll}1.7 & 04\end{array}$ & $\begin{array}{ll}1,5 & 03\end{array}$ \\
\hline$-1,00$ & $-1,6$ & $-2,3$ & $-4,0$ & $\mid-6,5$ & $\begin{array}{ll}3,0 & 02\end{array}$ & $\begin{array}{lll}8,6 & 07\end{array}$ & 4,3 \\
\hline
\end{tabular}

Tableau VIII. - Coefficient d'aberration chromatique principale des miroirs $O M E$.

[Principal chromatic aberration coefficient of the OME mirrors.]

\begin{tabular}{|c|c|c|c|c|c|c|c|}
\hline$v_{3 /}$ TY & OME 90 & OME 85 & OME 80 & OME 75 & OME 70 & OME 65 & OME 60 \\
\hline$-0,15$ & $\begin{array}{ll}2.0 & 01\end{array}$ & 7,2 & 7,5 & 8,001 & $\begin{array}{ll}1,9 & 02\end{array}$ & $\begin{array}{ll}1,8 & 02\end{array}$ & $\begin{array}{ll}1,2 & 02\end{array}$ \\
\hline$-0,20$ & $\begin{array}{ll}1,2 & 01\end{array}$ & 8,4 & $\begin{array}{ll}-1,1 & 01\end{array}$ & $\begin{array}{ll}-1,2 & 01\end{array}$ & $\begin{array}{ll}5,3 & 03\end{array}$ & $\begin{array}{ll}5,7 & 03\end{array}$ & $2,0 \quad 04$ \\
\hline$-0,25$ & 9,8 & 8.4 & $-3,2$ & $\begin{array}{ll}-5,3 & 01\end{array}$ & $\begin{array}{ll}1,4 & 03\end{array}$ & $\begin{array}{ll}9,8 & 01\end{array}$ & $\begin{array}{lll}9,5 & 01\end{array}$ \\
\hline$-0,30$ & $\begin{array}{ll}1,1 & 01\end{array}$ & 8,5 & 3,2 & $\begin{array}{ll}-2,5 & 01\end{array}$ & $\begin{array}{ll}-2,3 & 03\end{array}$ & $\begin{array}{ll}4,1 & 02\end{array}$ & $\begin{array}{ll}2,4 & 01\end{array}$ \\
\hline$-0,35$ & $\begin{array}{ll}1,4 & 01\end{array}$ & 9,6 & 6,0 & $-7,6$ & $\begin{array}{ll}-1,8 & 02\end{array}$ & $\begin{array}{ll}-5,6 & 01\end{array} \mid$ & 6,6 \\
\hline$-0,40$ & $\begin{array}{ll}2,3 & 01\end{array}$ & $\begin{array}{ll}1,3 & 01\end{array}$ & 7,9 & $5,2-01$ & $\begin{array}{ll}-4,9 & 01\end{array}$ & $\begin{array}{ll}-3,9 & 01\end{array}$ & $-5,5$ \\
\hline$-0,45$ & $\begin{array}{ll}5,2 & 01\end{array}$ & $\begin{array}{ll}1,9 & 01\end{array}$ & $\begin{array}{ll}1,1 & 01\end{array}$ & 4,8 & $\begin{array}{ll}-1,6 & 01\end{array}$ & $\left|\begin{array}{ll}-6,2 & 03\end{array}\right|$ & $\begin{array}{ll}-1,9 & 01\end{array}$ \\
\hline$-0,50$ & $\begin{array}{ll}2,0 & 02\end{array}$ & $\begin{array}{ll}3,7 & 01\end{array}$ & $\left|\begin{array}{ll}1,6 & 01\end{array}\right|$ & 7,9 & -3.7 & $\left|\begin{array}{ll}-1,8 & 02\end{array}\right|$ & $\mid \begin{array}{ll}-5,6 & 01\end{array}$ \\
\hline$-0,55$ & $\begin{array}{ll}6,2 & 07\end{array}$ & $\begin{array}{ll}9,9 & 01\end{array}$ & $\begin{array}{ll}2,6 & 01\end{array}$ & $\begin{array}{ll}1,2 & 01\end{array}$ & 2,9 & $\left|\begin{array}{ll}-4,6 & 01\end{array}\right|$ & $\mid \begin{array}{ll}-2,2 & 02\end{array}$ \\
\hline$-0,60$ & $2,2 \quad 02$ & $\begin{array}{ll}8,6 & 02\end{array} \mid$ & $\begin{array}{ll}5,4 & 01\end{array}$ & $\left|\begin{array}{ll}1,9 & 01\end{array}\right|$ & 7,6 & $\mid \begin{array}{ll}-1,5 & 01\end{array}$ & $\left|\begin{array}{ll}-1,3 & 04\end{array}\right|$ \\
\hline$-0,65$ & $\begin{array}{lll}5,7 & 01\end{array}$ & $\begin{array}{ll}1,1 & 03\end{array}$ & $\begin{array}{ll}1,9 & 02\end{array}$ & $\begin{array}{ll}3,3 & 01\end{array}$ & $\begin{array}{ll}1,3 & 01\end{array}$ & $-2,8$ & $\left|\begin{array}{ll}-4,7 & 02\end{array}\right|$ \\
\hline$-0,70$ & $\begin{array}{ll}2,7 & 01\end{array}$ & $\begin{array}{ll}1,2 & 02\end{array}$ & $\begin{array}{ll}7,7 & 03\end{array}$ & $\begin{array}{ll}7,9 & 01\end{array}$ & $\begin{array}{ll}2,2 & 01\end{array}$ & 5,2 & $\left|\begin{array}{ll}-8,7 & 01\end{array}\right|$ \\
\hline$-0,75$ & 1,6 ol & $\begin{array}{ll}4,1 & 01\end{array}$ & $\begin{array}{ll}4,2 & 02\end{array}$ & $\left|\begin{array}{ll}3,1 & 03\end{array}\right|$ & $\left|\begin{array}{ll}4,1 & 01\end{array}\right|$ & $\begin{array}{lll}1,2 & 01\end{array}$ & $\begin{array}{ll}-2,9 & 01\end{array}$ \\
\hline$-0,80$ & $\begin{array}{ll}1,1 & 01\end{array}$ & $\begin{array}{ll}2,3 & 01\end{array}$ & $\mid \begin{array}{ll}8,7 & 01\end{array}$ & $\mid \begin{array}{ll}1,9 & 05\end{array}$ & 9,201 & $\begin{array}{ll}2,3 & 01\end{array}$ & $-7,1$ \\
\hline$-0,85$ & 7,1 & $\begin{array}{lll}1,5 & 01\end{array}$ & $\begin{array}{ll}3,6 & 01\end{array}$ & $\left|\begin{array}{ll}3,3 & 02\end{array}\right|$ & $\begin{array}{ll}3,8 & 02\end{array}$ & $\begin{array}{lll}4,3 & 01\end{array}$ & 6,9 \\
\hline$-0,90$ & 6,3 & $\begin{array}{ll}1,0 & 01\end{array}$ & $\begin{array}{ll}2,1 & 01\end{array}$ & $\left|\begin{array}{ll}8,7 & 01\end{array}\right|$ & $\begin{array}{ll}1,5 & 06\end{array}$ & 9,6 of & $\begin{array}{ll}2,1 & 01\end{array}$ \\
\hline$-0,95$ & 5,1 & 7,9 & $\begin{array}{ll}1,4 & 01\end{array}$ & $\left|\begin{array}{ll}4,0 & 01\end{array}\right|$ & $\begin{array}{ll}3,9 & 02\end{array}$ & 3,6 of & 4,5 on \\
\hline$-1,00$ & 4,3 & 6,4 & $\begin{array}{ll}1,1 & 01\end{array}$ & $\begin{array}{ll}2,4 & 01\end{array}$ & $\begin{array}{ll}9,6 & 01\end{array}$ & $\begin{array}{ll}3,2 & 04\end{array}$ & $\mid \begin{array}{ll}9,7 & 01\end{array}$ \\
\hline
\end{tabular}




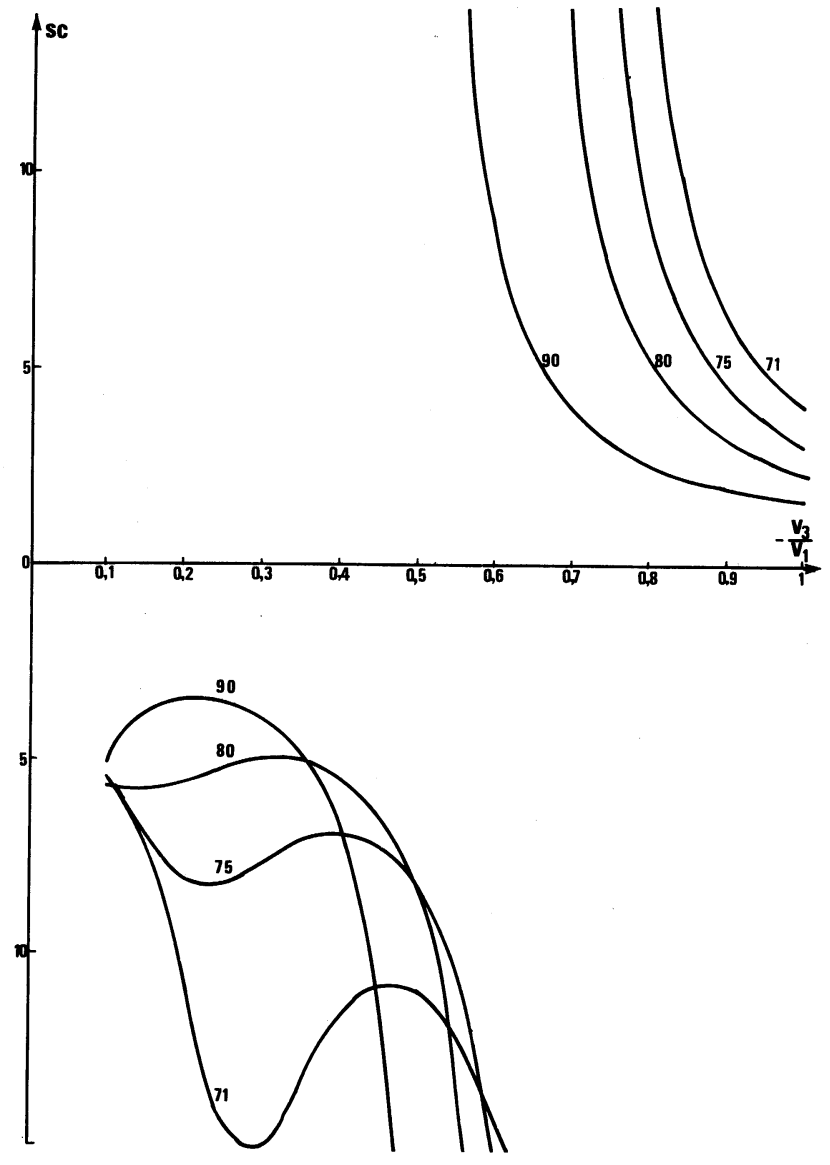

Fig. 4. - Variation du rayon de courbure $R$ de quelques miroirs du type OMD en fonction du rapport $V_{3} / V_{1}$ des potentiels extrêmes.

[The curvature radius $R$ of some OMD mirrors as a function of the ratio $V_{3} / V_{1}$ of outer cylinder potentials.]

valeur de $V_{2}$ diffère peu de celle de $V_{3}$. Au fur et à mesure que l'écart relatif entre $V_{2}$ et $V_{3}$ augmente, c'est-à-dire que $R_{\mathrm{d}}$ diminue, l'allure des courbes subit des évolutions de plus en plus importantes.

On est ainsi amené à considérer deux types de courbes.

- Pour les courbes des miroirs OMD (90 à 70) et des miroirs OME (90 à 75) on distingue dans la gamme des valeurs $V_{3} / V_{1}$ étudiées une zone où le miroir est convergent et une zone où il est divergent.

- Pour les courbes des miroirs OME (70 à 60)

l'évolution est plus considérable et l'on observe alors dans la même gamme de $V_{3} / V_{1}$ quatre zones du point de vue de la convergence.

Ces résultats sont aisément explicables, quand on examine la distribution du potentiel sur l'axe et en particulier le signe de la dérivée seconde responsable de l'effet convergent ou divergent.

3) Pour ces différents miroirs on remarque la présence de zones où la variation du rayon avec $V_{3} / V_{1}$ est peu importante. On peut y observer éventuellement un ou deux extréma, qui ont des valeurs diverses selon les tensions envisagées.

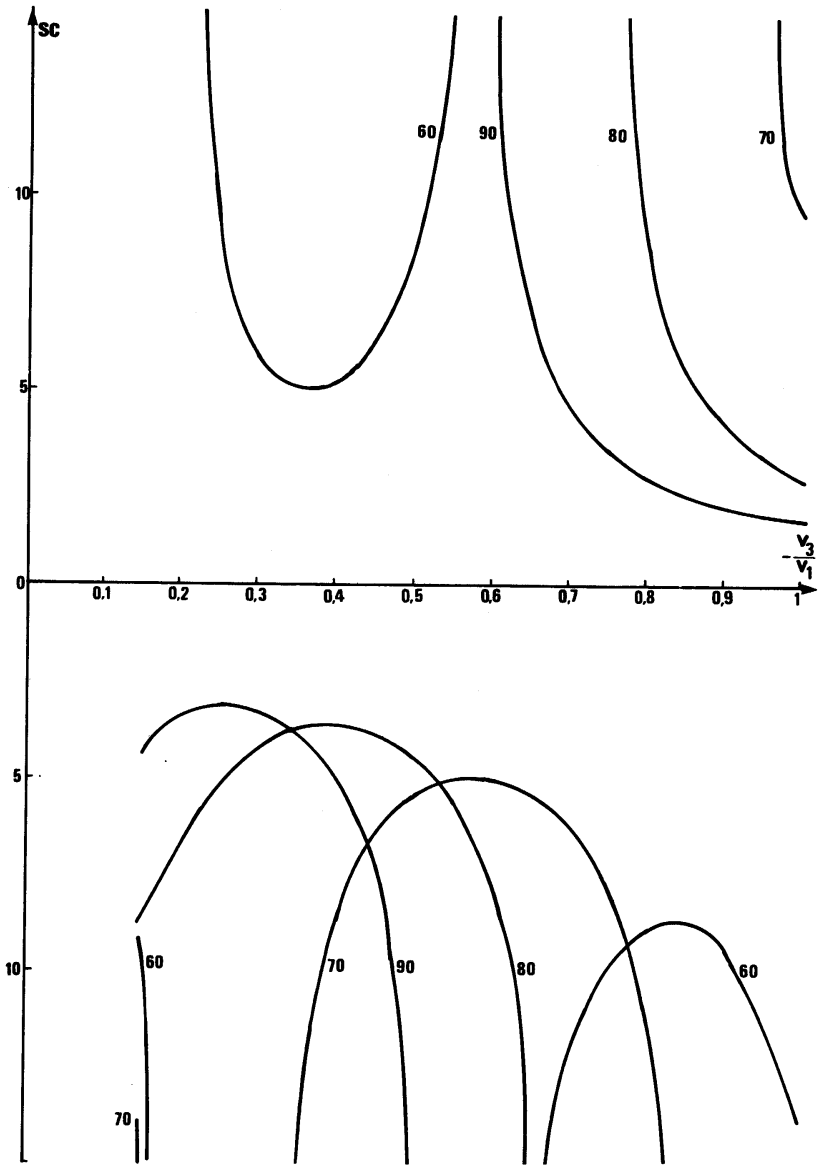

Fig. 5. - Variation du rayon de courbure $R$ de quelques miroirs du type OME en fonction du rapport $V_{3} / V_{1}$ des potentiels extrêmes.

[The curvature radius $R$ of some OME mirrors as a function of the ratio $V_{3} / V_{1}$ of outer cylinder potentials.]

4) Dans ces zones à faibles variations, les coefficients d'aberration sphérique ou chromatique ont la particularité d'être également faibles. De plus au voisinage des extréma, ces coefficients présentent des particularités :

soit ils changent de signe en s'annulant,

soit leur valeur absolue passe par un minimum.

Quand on utilise les propriétés focalisatrices des miroirs, le fonctionnement dans une telle zone est donc intéressant. Ces propriétés avaient déjà été mentionnées lors de l'étude des miroirs bicylindriques' [2]. Signalons tout particulièrement les miroirs OMD fonctionnant dans la zone $V_{3} / V_{1}$ voisine de $-0,30$ où il est possible d'annuler ou de rendre très faibles les coefficients d'aberration sphérique et chromatique et d'obtenir le rayon de courbure souhaité en agissant sur le potentiel intermédiaire $V_{2}$.

5. Conclusion. - Les différents miroirs ont été étudiés sur une gamme étendue des rapports $V_{3} / V_{1}$ des potentiels extrêmes. De multiples possibilités de réaliser une même convergence sont ainsi offertes. 
Ce choix permet tout d'abord de satisfaire à certaines contraintes externes quant au positionnement des éléments cardinaux du miroir. Ensuite, s'il existe encore une possibilité de choix, on retiendra la solution améliorant le plus les qualités optiques importantes pour le système à réaliser.

\section{Bibliographie}

[1] Kel'Man, V. M., Fedulina, L. V. et Yakushev, E. M., $Z h$. Tekh. Fiz. 41 (1971) 1489, Zh. Tekh. Fiz. 41 (1971) 1832

[2] Berger, C. et Baril, M., Revue Phys. Appl. 14 (1979) 783.

[3] Dard, J. et Berger, C., J. Microsc. Spectrosc. Electron. 4 (1979) 361

[4] Berger, C., Lambinet, M. et Baril, M., J. Microsc. Spectrosc. Electron. 4 (1979) 333
[5] Berger, C., Lambinet, M. et Baril, M., Revue Phys. Appl. 15 (1980) 61

[6] Durand, E., Electrostatique, tome 1 (Edition Masson et Cie) 1966.

[7] Weber, C., Philips Tech. Rev. 24 (1963) 130

[8] Berger, C. et Baril, M., Revue Phys. Appl. 13 (1978) 271. 